\title{
What Processes Know: Definitions and Proof Methods
}

(Preliminary Version)

by

\author{
Shmuel Katz and Gadi Taubenfeld \\ Computer Science Department \\ Technion, Haifa 32000 \\ Israel
}

\begin{abstract}
The importance of the notion of knowledge in reasoning about distributed systems has been recently pointed out by several works. It has been argued that a distributed computation can be understood and analyzed by considering how it affects the state of knowledge of the system. We show that there are a variety of definitions which can reasonably be applied to what a process can know about the global state. We also move beyond the semantic definitions, and present the first proof methods for proving knowledge assertions. Both shared memory and message passing models are considered.
\end{abstract}

\section{INTRODUCTION}

In this paper we will show how to prove knowledge assertions about both distributed and shared memory parallel programs. Before describing the proof methods we consider the fundamental question of "what is the meaning of knowledge in concurrent programs?" Put another way, what does it mean for (an observer of) a process to know something? Other tempting questions immediately arise: What does a process always know? What does a process know whenever it terminates? What does it know at a certain moment during a given execution?
A number of successful attempts have been made in order to formally capture various notions of knowledge [HM1,HM2,FHV,Le]. Some other recent works are directly connected to a concurrent programming environment. In [CM] a characterization is described of the minimum information flow necessary for a process to gain or lose knowledge about the system. The results are applied for proving lower bounds on the number of messages required to solve certain problems. In $[\mathrm{HF}]$ a formal model that relates the concepts of knowledge, action and communication in distributed systems is presented. An impossibility proof for the well known coordinated-attack problem is presented. In [PR] a connection between distributed systems and the logic of knowledge is established. A set of axioms which this knowledge must obey is presented.

We first motivate our approach to knowledge. Consider a concurrent program which is frozen at some point during its execution. Let $b$ stand for a predicate defined on the global state of that program. Now, suppose you are interested in knowing whether predicate $b$ is true when the program is suspended. For this task, you are allowed to use certain global information which is assumed to be known a priori. Such infornation might be, for example, an invariant of the program, certain properties of the processes, or properties of the model. In addition it is possible that you have access to 
the following local information about the behavior of process $i$.

case one: no additional information.

case two: the control position of process $i$.

case three: the complete visible state of process $i$.

case four: the complete history of process $i$.

For a distributed model, a process' visible state is identical to its local state. In the shared variable model, a process' visible state refers to all variables it can test. The history of a process means the sequence of all events in which it has participated.

We say that process $i$ knows $b$ when the program is suspended, if using the above information it is possible to prove that $b$ holds. Thus a process' knowledge is always defined with respect to some given information. Note that the global information is static, in the sense that it does not change during an execution, while the local information is dynamic. We call the available global and local information, the knowledge assumption.

In this paper we assume the (entire) text of the program and its input specification as the a priori global information of a process. The opposite extreme would be to assume no global information. This case is of little interest since extremely limited knowledge is possible when even the properties of the model are not known a priori. A less extreme case, where nothing is assumed about some of the processes, is of greater interest. A possible application for such a case is the Byzantine Generals Problem, where the protocol which a faulty Byzantine process is following is not available. However, it follows from [LSP] that in order for the processes to know the agreed value, it must be part of the global information that over two thirds of the processes are following a protocol which is known. An intermediate level is also possible, in which only certain properties of the program are assumed to be known a priori. This level would be appropriate during program development. In [HF] it is not assumed that the text is given.
Given our assumption of maximal global information, the above four cases of local information are considered to define different types of knowledge. Cases one and four represent opposite extremes from process $i$ 's point of view. In case one, called invariant knowledge, no local information at all is available, and thus process $i$ may know only 'general things' like 'the program terminates' and all safety properties. In case four, called history knowledge, all possible local information is available, which of course, leads to the strongest type of knowledge. Numerous intermediate levels, where only a partial history is given, can be defined between those extremes. Examples are cases two and three, called location and state knowledge, respectively. Previous papers [CM,HF,PR] refer to (variants of) the concept of history knowledge. They all mention state knowledge as an additional option. Location knowledge refers to the knowledge of a process at a certain point for all possible computations. We establish in the sequel a connection between fundamental verification concepts such as proof outline, interference freedom and cooperation [AFR,OG] and the concepts of knowledge. A method for proving knowledge assertions of all types is presented.

\section{THE MODEL}

A concurrent program is a collection of a finite set of processes operating asynchronously, which may communicate with each other in one of the following ways, (a) asynchronously using send and receive, (b) synchronously, as in CSP, also using send and receive commands, or $(c)$ by setting shared variables.

An event is the basic unit in the behavior of a process. It describes a change in the environment during the process' execution. An event occurs on a process, by executing an atomic command of that process. An execution of an atomic command includes executing a skip or assignment command, a loop exit, evaluation of a boolean expression, or execution of a com- 
munication. ${ }^{1}$ The same atomic commands executed in different environments correspond to different events. A behavior of a process can be described as a finite sequence of events on that process. This is known as a trace $[B H R, M]$. A process is characterized by a (prefix-closed) set of all its possible traces. By generalizing the above, a concurrent program $P$ is defined. Assuming an interleaving semantics, we are only interested in the relative order in which events occur. No clock is assumed. A trace $t$ of $P$ is a finite sequence of events on processes of $P$ satisfying the conditions:

(1) Any subsequence of $t$ which consists of all events on a process which belongs to $P$, is a trace of that process.

(2) Asynchronous communication: for every event of 'receiving message $m$ ' there is a corresponding 'send message m' event which occurs earlier.

(2) Synchronous communication: for every event of 'send message $m$ ' there is a corresponding 'receive message m' event which occurs immediately after, i.e, no other event occurs between those events (those two events can be considered to be a single one).

A concurrent program is characterized as the set of all possible traces of the program.

A trace can also be seen as a transition from the initial state of the program into a unique state of the program, which in addition records all the 'history' of how it reached that state. Each such state contains two kinds of information: the exact values of the program variables and the control position of each process (the value of the program counter).

To be able to express the control position, we assume that all commands are uniquely labeled. By saying that (the control of) a process is after $c$, we mean that the command labeled $c$ was the last one to be executed in that process. In addition the labels

The language may also contain selection statments with local init $_{1}, \ldots$, init $t_{n}$ are used to label dummy commands placed at the beginning of each process. If (control of) process $i$ is after init $_{i}$, execution of process $i$ has not yet started. ${ }^{2}$

With each trace $t$ we associate a (trace) predicate $s_{t}$ which expresses the values of all the variables (including the control) after a computation described by $t$ took place. This predicate depends on a given initial state. For the rest of the paper let $l_{i}$ stand for a label of a command from process $p_{i}(i=1 . . n)$. Let $C$ be a function from traces into $n$-tuples of labels.

Definition: $C(t)=\left(l_{1}, \ldots, l_{n}\right)$ iff after a computation described by $t$ the control of processes $p_{1}, \ldots, p_{n}$ is after $l_{1}, \ldots, l_{n}$ respectively.

A location set $T_{\left(l_{1}, \ldots, L\right)}$ is defined to be a set of traces such that: $T_{\left(l_{1}, \ldots, l_{n}\right)} \equiv\left\{t \mid C(t)=\left(l_{1}, \ldots, l_{n}\right)\right\}$. Thus location set $T_{\left(1_{1}, \ldots, l_{0}\right)}$ describes the set of traces which leave the control of the program after $\left(l_{1}, \ldots, l_{n}\right)$. It easy to see that the number of such location sets is exactly the product of the number of labels in each process. They correspond to all possible positions of the control of the program during an execution.

For the rest of the paper, let $l c$ denote an $n$ tuple of labels $\left(l_{1}, \ldots, l_{n}\right)$. With each location set $T_{k}$ we associate a location predicate $S_{l c} . S_{k}$ is defined to be the disjunction of all $s_{t}$ such that $t$ belongs to $T_{k}$. If $T_{k c}$ is an empty set (i.e for no execution is the control after $l c$ ), then $S_{l c}$ is false. Assume that the control of a program is after $l c$. The state of a specific execution at this point refers to the exact values of all program (and environment) variables. The location predicate $S_{l c}$ at this point refers to all possible values of the variables, without committing to a certain execution. $S_{k}$ is actually the strongest predicate which is true when the control is after $l c$.

nondeterminism, but this will not be atomic.

${ }^{2}$ In case that $c$ and $d$ denote two different labels of distinct commands belonging to the same process, then the assertion after (c) ^ after (d) equals false. 
Example 1: Consider the (shared variable) pro$\operatorname{gram} P::\left[P_{1} \| P_{2}\right]$ where:

$$
\begin{aligned}
& P_{1}:: \text { end }_{1}: x:=x+1 \\
& P_{2}: \text { end } 2: x:=2^{*} x
\end{aligned}
$$

Assume that the initial state is $x=2$.

The possible events are: (1) end 1 executes first, (2) end ${ }_{2}$ executes first, (3) end executes second, (4) end $_{2}$ executes second.

The predicates associated with the traces $\langle>,<1\rangle,<2\rangle,<2,3\rangle,<1,4>$ are:

$s_{<>} \equiv x=2 \wedge$ after (init 1, init $\left._{2}\right)$,

$s_{<1\rangle} \equiv x=3 \wedge$ after $\left(\right.$ end $_{1}$, , init $\left._{2}\right)$,

$s_{<2>} \equiv x=4 \wedge$ after $\left(\right.$ init $_{1}$, end $_{2}$ ),

$s_{<2,3\rangle} \equiv x=5 \wedge$ after $\left(\right.$ end $_{1}$, end $\left._{2}\right)$,

$s_{<1,4\rangle} \equiv x=6 \wedge$ after $\left(\right.$ end $_{1}$, end $\left.d_{2}\right)$, respectively.

The possible location sets are:

$T_{\left(\text {init }_{1}, \text { nit }_{2}\right)} \equiv\{<>\}, T_{\left(e n d_{1}, \text { nit }_{2}\right)} \equiv\{<1>\}$,

$T_{\left(\text {init }_{1}, e n d_{2}\right)} \equiv\{<2>\}, T_{\left(\text {end }_{1}, e n d_{2}\right)} \equiv\{<2,3>,<1,4>\}$.

The possible location predicates are:

$S_{\left(\text {init }_{1}, \text { init }_{2}\right)} \equiv x=2 \wedge$ after (init ${ }_{1}$, init $\left.{ }_{2}\right)$,

$S_{\left(\text {end }_{1}, \text { init }_{2}\right)} \equiv x=3 \wedge$ after $\left(\right.$ end $_{1}$, init $\left._{2}\right)$,

$\left.S_{\left(\text {init }_{1}, \text { end }\right.}\right) \equiv x=4 \wedge$ after (init ${ }_{1}$, end $\left._{2}\right)$,

$S_{\left(e n d_{1}, e n d_{2}\right)} \equiv((x=5) \vee(x=6)) \wedge$ after $\left(\right.$ end $_{1}$, end $\left._{2}\right)$.

We will also assume that each process $i$ has a local history named $h_{i}$. The history will presumably be accumulated in a variable which records the sequence of all events in which process $i$ has participated. As mentioned in $[\mathrm{HF}]$ "This is certainly not a reasonable assumption", because it assumes that a process has unbounded storage space to record its history. A trace $t$ matches $h_{i}$ (match $\left.\left(t, h_{i}\right)\right)$ iff $t_{i}$, the subsequence of $t$ which consists of all events on process $i$, equals $h_{i}$. Similarly $h_{i}$ matches location set $T_{l c}$ iff it matches with some trace belonging to $T_{l c}$.

A history location predicate $-S h_{l c}$ - associated with location set $T_{k c}$ and history $h$, is defined as the disjunction of all (trace) predicates $s_{t}$ such that $t$ belongs to $T_{l k}$ and match $(t, h)$. If no such traces exist then
$S h_{l c} \equiv f a l s e$. The predicate $S h_{l c}$ is, by definition, the strongest predicate known to be true after $l c$ when only $h$ is known.

Example: If the local history $h$ of $P_{1}$ was empty (no events have yet occurred), then that history matched the first and third traces given above. The possible history location predicates in that case are:

$S h_{\left(\text {init }_{1}, \text { init }_{2}\right)} \equiv x=2 \wedge$ after $\left.\left(\text { init }_{1}, \text {, }_{\text {init }}\right)_{2}\right)$,

$S h_{\left(\text {end }_{1}, \mathrm{init} \mathrm{t}_{2}\right)} \equiv$ false,

$\left.S h_{\left(\text {init }_{1}, \text { end }\right.}\right) \equiv x=4 \wedge$ after $\left(\right.$ init $_{1}$, end $\left._{2}\right)$,

$S h_{\left(e n d_{1}, e n d_{2}\right)} \equiv$ false.

\section{DEFINITION OF KNOWLEDGE}

The (global) trace which corresponds to the execution up to any moment, belongs to exactly one location set (called the actual location set). Informally, we will say that process $i$ knows $b$ iff $b$ is true in all location sets that process $i$ considers possible candidates to be the actual one.

In the first case where no additional local information is given, process $i$ must consider all location sets which are possible under the global a priori information. When, in case two, process $i$ knows the exact position of its control, the possible location sets reduce to those for which $l_{i}$ corresponds to $i$ 's actual position. With the additional assertion giving the exact visible state of process $i$, in case three, the above possible location sets reduce to those where the location predicate of the location sets does not contradict the assertion about the actual visible state. Finally, with the history of process $i$, only those location sets which match that local history are possible.

For now, we restrict the language so that no knowledge operator appears within the scope of another. Let $c$ be a label of a command in process $i, s$ be an assertion describing a visible state of process $i$ (including the control of process $i$ ), and $h$ be a history of process $i$. 


$\begin{array}{ll}\quad P::\left[P_{1} \| P_{2}\right] \text { where: } & \\ P_{1}:: & P_{2}:: \\ a:[a 1: \text { true } \rightarrow x:=1 \rrbracket a 2: \text { true } \rightarrow x:=2] ; & d:[\text { true } \rightarrow y:=1 \square \text { true } \rightarrow y:=2 \square \text { true } \rightarrow y:=3] ; \\ b: P_{2} ! x ; & e: P_{2} ? z ; \\ c: P_{2} ? x & f: P_{2} !(y+z)\end{array}$

Figure 1. Simple CSP Program.

The notations $K I_{i}(b), K L_{i}^{c}(b), K S_{i}^{s}(b), K H_{i}^{h}(b)$ are used for: process $i$ knows $b$, process $i$ knows $b$ after $c$, process $i$ knows $b$ with $s$, process $i$ knows $b$ with $h$, respectively. Recall that $l c$ is an $n$-tuple of labels.

Definition :

(1) Invariant knowledge:

$$
K I_{i}(b) \text { iff } \forall l c . S_{l c} \rightarrow b .
$$

(2) Location knowledge:

$$
K L_{i}^{c}(b) \text { iff } \forall l c .\left(S_{l c} \wedge \text { after }(c)\right) \rightarrow b .
$$

(3) State knowledge:

$$
K S_{i}^{s}(b) \text { iff } \forall l c .\left(S_{l c} \wedge s\right) \rightarrow b .
$$

(4) History knowledge:

$$
K H_{i}^{h}(b) \text { iff } \forall l c . S h_{l c} \rightarrow b .{ }^{3}
$$

Let $K_{i}$ denote all knowledge types. The following properties are over all labels, visible states and histories.

\section{Properties:}

(1) $\left(K_{i}(b) \wedge\left(b \rightarrow b^{\prime}\right)\right) \rightarrow K_{i}\left(b^{\prime}\right)$.

(2) $K_{i}(b) \wedge K_{i}\left(b^{\prime}\right)$ iff $K_{i}\left(b \wedge b^{\prime}\right)$.

(3) $\left(K_{i}(b) \vee K_{i}\left(b^{\prime}\right)\right) \rightarrow K_{i}\left(b \vee b^{\prime}\right)$.

(4) $\left.\left(K_{i}\left(b \rightarrow b^{\prime}\right) \wedge K_{i}(b)\right) \rightarrow K_{i}\left(b^{\prime}\right)\right)$.

(5) $K L_{i}^{c}($ after (c)).

(6) $K S_{i}^{s}(s)$.

$K H_{i}^{h^{3}}(b)$ iff $\forall t \cdot$ alternative $t_{t}^{\text {equivalent }}$
(7) $\left(K L_{i}^{c}(b) \wedge(s \rightarrow\right.$ after $\left.(c))\right) \rightarrow K S_{i}^{s}(b \wedge s)$.

We give a proof of property (7):

(1) $K L_{i}^{c}(b)$.

[Assumption]

(2) $s \rightarrow$ after (c).

[Assumption]

(3) $\forall l c \cdot\left(S_{l c} \wedge a f t e r(c)\right) \rightarrow b$

(4) $\forall l c \cdot\left(S_{l c} \wedge s\right) \rightarrow b$.

[(1), Def. of location knowledge]

(5) $K S_{i}^{s}(b)$.

[(4), Def. of state knowledge]

(6) $K S_{i}^{s}(b \wedge s)$.

[(5), properties $2 \& 6]$

[]

Example 2: Consider the (CSP) program, shown in figure 1. Let $s$ describe a local state of $P_{1}$, $s \equiv\{x=3 \wedge$ after $(c)\}$, Let $h$ be a history of $P_{1}$. Assume that it implies that the current local state is the one described by $s$, and that the first event on $P_{1}$ occurred by executing command $a 1$. The following knowledge assertions are then true:

(1) $K I_{1}(y=1 \vee 2 \vee 3 \vee$ Undefined $)$, (2) $K L_{1}^{c}(y=1 \vee 2 \vee 3)$,

(3) $K S_{1}^{s}(y=1 \vee 2)$. (4) $K H_{1}^{h}(y=2)$.

In the next section, we formally prove all these knowledge assertions.

\section{THE PROOF METHOD}

In this section a method for proving knowledge assertions about a program is presented. We initially establish a connection between location knowledge and various verification methods. The method for 
proving location knowledge assertions is then developed based on existing verification methods. For proving history knowledge assertions, an original method is developed, based on the new notion of history outline. For now, we restrict a process to know only predicates which do not contain temporal operators. In the future we intend to relax that restriction.

From the variety of verification methods available [B], we choose two proof systems [AFR,OG] which we consider representative. Both systems are designed to prove partial correctness, one for CSP and the other for a shared variable model. A stronger system, such as [OL], which can be used for proving liveness properties, should be considered when temporal operators are incorporated.

In each of the two systems, a partial correctness proof of a program is done in two stages: (1) separate proofs are constructed in isolation for each component process, (2) the separate sequential proofs are combined by showing that they are interference -free [OG] or cooperate [AFR].

The separate proofs, in the first stage, are presented by a proof outline in which each statement of a process is preceded and followed by a corresponding assertion. Next, in the second stage it is proved that the possible interactions do not invalidate the sequential proofs. In the shared variable model, it is necessary to show that the assertions used in the proof outline of each process are left invariantly true under parallel execution of the other processes. This is done by proving interference-freedom. In the distributed model, when establishing the separate proofs, a process 'guesses' the value its parameters will receive upon communication. When the proofs are combined, these guesses have to be checked for consistency using the cooperation test. In order to combine the separate sequential proof outlines, a parallel composition (meta) rule is used.

Both proof systems use additional auxiliary variables for the correctness proof. In [AFR] the notion of brackets is introduced, in order to delimit the sections within which an invariant need not necessarily hold. Here we assume that an interleaving can occur between every two atomic statements, so that all possible states after an atomic action must be considered in deciding what is known. For this reason, a bracketed section is restricted to contain exactly one basic command (i.e a command which does not refer to auxiliary variables). This does not affect the completeness as is shown in [Ap]. An await statement (for the shared-memory model) or a bracketed section is considered as an atomic action. For later reference, we denote a program $P$ with additional auxiliary variables by $P^{\prime}$.

In the proof outline for process $i$, post $\left(l_{i}\right)$ stands for the post assertion of a command labeled by $l_{i}$. A global invariant which may be used for the proof is denoted by $I$, while post $\left(l_{1}, \ldots, l_{n}\right)$ is an abbreviation for $\bigwedge_{i=1}^{n} \operatorname{post}\left(l_{i}\right) \wedge I \wedge \operatorname{after}\left(l_{1}, \ldots, l_{n}\right)$.

\subsection{LOCATION KNOWLEDGE}

We now present a proof method for proving location knowledge assertions. First, a theorem which links the definition of location knowledge and that of interference free (cooperating) proof outlines is formulated. Then we give the proof method itself.

Lemma 1: Assume a program with a locally correct sequential proof outline for each process. If the proof outlines of the individual processes are interference-free (cooperate) then for every label $l_{i}$ of process $i(i=1 . . n), K L_{i}{ }^{i}\left(\right.$ post $\left.\left(l_{i}\right)\right)$, i.e, process $i$ knows (location knowledge) post $\left(l_{i}\right)$ after $l_{i}{ }^{4}$

Proof: Assume to the contrary that for some $l_{i}$, $K L_{i}^{h}$ (post $\left.\left(l_{i}\right)\right)$ does not hold. This means that there exists a trace $t$ such that $C(t)=\left(l_{1}, \ldots, l_{n}\right)$ and its associated predicate $s_{t}$ does not imply post $\left(l_{i}\right)$, which immediately contradicts the soundness [Ap, Ow] of the proof systems.

${ }^{4}$ Here and afterwards only executions which start in an initial state satisfying post ( init $_{1}, \ldots$, init $_{n}$ ) are considered. 
Remark: Let us define the assertions of (annotated) process $i$ as a correct sequential location outline iff for every label $l_{i}$ of process $i, K L_{i}{ }^{h}$ (post $\left(l_{i}\right)$ ). We claim that in order to prove partial correctness of a program, it is sufficient to establish such a location outline for each process, and then apply to them a (modified) parallel composition (meta) rule without further use of interference-freedom, cooperation or any other test. From the above lemma and claim the interferencefreedom test and the cooperation test are viewed as providing sufficient (but not necessary!) conditions for a correct sequential proof outline to be also a location outline.

For the rest of the section, we refer to a program with correct interference-free (cooperating) proof outlines. In fact, it is sufficient to have correct location outlines.

Corollary 1.1: For any location set $T_{l c}$ its associated predicate $S_{l c}$ implies post (lc).

Proof: The location predicate $S_{l c}$ is the strongest predicate true after $l c$, while it follows from Lemma 1 that post $(l c)$ is a true predicate after $l c$.

Theorem 1: If $c$ is a label of process $i$, and $\Phi(c)$ is the disjunction of all assertions post $\left(l_{1}, \ldots, l_{n}\right)$ such that $l_{i} \equiv c$, then $K L_{i}^{c}(\Phi(c))$.

\section{Proof:}

(1) $\forall l c .\left(S_{l c} \wedge\right.$ after $\left.(c)\right) \rightarrow($ post $(l c) \wedge$ post $(c))$.

[From corollary 1.1]

(2) $\forall l c$.(post $(l c) \wedge$ after $(c)) \rightarrow \Phi(c)$. [Def. of $\Phi(c)$ ]

(3) $\forall l c .\left(S_{l c} \wedge\right.$ post $\left.(c)\right) \rightarrow \Phi(c)$.

(4) $K L_{i}^{c}(\Phi(c)) . \quad$ [(3), Def. of location knowledge] []

The preceding theorems refer to a given program which, as mentioned, may contain auxiliary variables. A rule similar to the usual rule [OG] for removing such variables is used, to refer back to the original program (i.e without auxiliary variables).
Awxiliary Variables rule:

Let $\mathrm{AV}$ be a set of variables such that $x \in \mathrm{AV}$ implies $x$ appears in $P^{\prime}$ only in assignments $y:=t$, where $y \in \mathrm{AV}$. Then if $\Psi$ does not contain free any variables from $\mathrm{AV}, P$ is obtained from $P^{\prime}$ by deleting all assignments to variables in $A V$, and processes $i$ and $i$ are corresponding processes belonging to $P$ and $P^{\prime}$ respectively,

$$
\frac{K L_{i}^{c}(\Psi)}{K L_{i}^{c}(\Psi)}
$$

Following is a method for proving location knowledge assertions. Suppose it is to be proved that $K L_{i}^{c}(b)$ is true for a given program $P$. The proof goes as follows:

(1) Using one of the mentioned proof systems [AFR,OG] a "strong enough" correctness proof is established for $P^{\prime}$.

(2) Assertion $\Phi(c)$, as defined above, is constructed. [by theorem $1 K L_{i^{\prime}}^{c}(\Phi(c))$ ].

(3) It is proved that $\Phi(c)$ implies $b$. [by property $1 K L_{i^{\prime}}^{c}(b)$ ].

(4) By applying the auxiliary variables rule, $K L_{i}^{c}(b)$ is proved.

Theorem 2 (completeness): If $K L_{i}^{c}(b)$ is true then it can be proved to be true.

Proof: Recall that $b$ is asserted to be nontemporal, and not to include knowledge assertions. It must be shown that if $K L_{i}^{c}(b)$ is true, then (in step(1)) a "strong enough" correctness proof can indeed be established. From the fact that the proof systems are complete [Ap,Ow], it follows that it is possible (although not practical) to establish a correctness proof in which for any $n$-tuple $\left(l_{1}, \ldots, l_{n}\right)$, post $\left(l_{1}, \ldots, l_{n}\right)$ is the strongest post assertion possible. Such a correctness proof is always "strong enough" [].

Example 3: Recall example 2. We prove $K L_{1}^{c}(y=1 \vee 2 \vee 3)$ 


$$
\begin{array}{lc}
P_{1}^{\prime}:: & P_{2}^{\prime}:: \\
\{i=0\} & \{j=0\} \\
a:[\text { true } \rightarrow x:=1 \square \text { true } \rightarrow x:=2] ; & d:[\text { true } \rightarrow y:=1 \text { \true } \rightarrow y:=2 \square \text { true } \rightarrow y:=3] \\
\{(x=1 \vee 2) \wedge i=0\} & \{(y=1 \vee 2 \vee 3) \wedge j=0\} \\
b:<P_{2} ! x ; i:=1> & e:<P_{2} ? z ; j:=1> \\
\{i=1\} & \{(y=1 \vee 2 \vee 3) \wedge(z=1 \vee 2) \wedge j=1\} \\
c:<P_{1} ? x ; i:=2> & f:<P_{2} !(y+z) ; j:=2> \\
\{(x=2 \vee 3 \vee 4 \vee 5) \wedge i=2\} & \{(y=1 \vee 2 \vee 3) \wedge(z=1 \vee 2) \wedge j=2\} \\
&
\end{array}
$$

Figure 2.

Proof Outline.

step 1: To verify the program, two auxiliary variables $i, j$ are used. The proof outline for the bracketed program $P^{\prime}$ is shown in figure 2 .

step 2: $\Phi(c) \equiv$ post $\left(c\right.$, init $\left._{2}\right) \vee$ post $(c, d) \vee$ post $(c, e) \vee$ post $(c, f)$ $\equiv$ false $\vee$ false $\vee$ false $\vee$ $((x=2 \vee 3 \vee 4 \vee 5) \wedge i=2 \wedge(y=1 \vee 2 \vee 3)$ $\wedge(z=1 \vee 2) \wedge j=2 \wedge I \wedge$ after $(c, f))$.

step 3: $\Phi(c)$ implies $y=1 \vee 2 \vee 3, \quad$ by property 1 $K L_{1}^{c} \cdot(y=1 \vee 2 \vee 3)$.

step 4: By applying the auxiliary variables rule, $K L_{1}^{c}(y=1 \vee 2 \vee 3) \quad$ ].

It is easy to see how the method is applied for proving state knowledge assertions as well. Let $\Psi$ be an assertion describing some location knowledge (of process $i$ ) after $c$ (proved using the above method), and for some computation let $s_{j}$ be an assertion describing the visible state of process $i$ after $c$. By property (7), $\Psi \wedge s_{i}$ is state knowledge of process $i$ after $c$ for that computation. This method is not strong enough and in the next section we show a better way for proving state knowledge assertions.

Example: Recall example 2 . We prove $K S_{1}^{s}(y=1 \vee 2)$. Let $\Psi \equiv(z=1 \vee 2) \wedge x=y+z$.
- $\Phi(c)$ implies $\Psi$. By property $1 K L_{1}^{c_{1}}(\Psi)$.

- By applying the auxiliary variable rule, $K L_{1}^{c}(\Psi)$.

- By property $4, K S_{1}^{s}(\Psi \wedge s) . \quad(s \equiv\{x=3 \wedge$ after $(c)\})$

- $\Psi \wedge s$ implies $y=1 \vee 2$; By property $1 K S_{1}^{s}(y=1 \vee 2)$. [].

\subsection{HISTORY KNOWLEDGE}

We now present a proof method for proving history knowledge assertions as well. First the notion of history outline is defined. It generalize the notion of cooperating proof outline (i.e a collection of sequential proof outlines which pass the cooperation test), and that of a location outline. Once this is done, a theorem which links the definition of history knowledge and that of a history outline is formulated. We conclude by giving the proof method itself. We restrict ourselves to the distributed model. It should be possible to apply the ideas presented in the sequel also to the shared variables model.

A history $h_{i}$ of process $i$ is expressed as a finite sequence of assertions describing i's local states $\left.<s_{0}, \cdots, s_{j}, s_{j+1}, \ldots, s_{n}\right\rangle$, where $s_{0}$ describes $i$ 's initial state and $s_{j+1}$ describes a successive state to $s_{j}$. We say that history $h_{i}$ implies after (c) iff $s_{n}$ implies after (c). 
Example 4: Consider example 2. A possible history for $P_{1}$ is $h_{1} \equiv<s_{0}, s_{1}, s_{2}, s_{3}, s_{4}>$, where:

$s_{0} \equiv\left\{\right.$ after (init $\left.\left.{ }_{1}\right)\right\}, s_{1} \equiv\{x=1 \wedge$ after $(a 1)\}$,

$s_{2} \equiv\{x=1 \wedge$ after $(a)\}, s_{3} \equiv\{x=1 \wedge$ after $(b)\}$,

$s_{4} \equiv\{x=3 \wedge$ after $(c)\}$.

We introduce the notion of a history outline (h.o.), which is a central concept to the proof method. Given history $h_{i} \equiv<s_{0}, \cdots, s_{n}>$, a h.o. is intuitively the assertions of an annotated program in which the information known from the given history, and its implications on the behavior of the rest of the processes, may be captured.

Assume an annotated program where each command $c: S$ of a process is preceded and followed by corresponding assertions pre $(c)$ and post $(c)$ respectively. We will define when those assertions form a correct h.o, with respect to a given history $h$. The definition is given using marking rules. Initially all assertions are assumed unmarked. Each assertion is expressed as a conjunction of conjuncts. A rule may allow marking a conjunct, and an assertion is marked when all its conjuncts are marked. The assertions form a correct h.o. with respect to history $h$ iff it is possible to mark them all.

The marking rules

Let post (c) denote the post assertion of a command labeled by $c$, and let $\xi$ denote one of its conjuncts. $\xi$ can be marked if it satisfies at least one of the following marking rules,

\# $\quad$ First rule: Let $P O$ stand for a possible cooperating proof outline established for the above bracketed program using the usual proof system ([AFR]). Let $p o(c)$ denote the post assertion belonging to $P O$, of the command labeled $c$.

* po $(c) \rightarrow \xi$. (From the previous section, this is equivalent to: $\quad * \quad K L_{i}^{c}(\xi)$ )

Remark: The various conjuncts marked by this rule do not necessarily refer to the same $P O$.
The rule assures that information expressed within an ordinary cooperating proof outline, can also be expressed within a h.o.. It follows immediately that a correct cooperating proof outline is also a correct h.o. with respect to any given history.

An Invariant is proven in the usual way, and can then be marked.

\# Second rule: Let $S$ be a command labeled by $c$.

* The weakest liberal pre-condition of $\xi$ and $S$ is implied by an already marked conjunct of pre (c).

This means that for any sequential Hoare rule, if all premises are marked, the consequent can be marked. Through the rule a marked conjunct can affect its local environment.

\# Third rule: Let $c$ be a label of the process $i$ to which the history $h$ is known $\left(h \equiv<s_{0}, \cdots, s_{n}>\right)$, and let $\Theta_{h}(c)$ denote the disjunction of all $s_{j}$ 's such that $s_{j} \rightarrow$ after $(c)$. (If for no $s_{j}$, $s_{j} \rightarrow$ after $(c)$ then $\Theta_{h}(c) \equiv$ false.)

* $\Theta_{k}(c) \rightarrow \xi$.

Through the rule, information from the history of a process is 'transferred' directly into the assertions of that process, with no further proof obligations.

\# Fourth rule (output): Let $c$ be a label of an output command $c: P_{j}$ lexpr. Let Inp be the set of all labels of input commands which semantically match ${ }^{5}$ with that output command. For each input command $d: P_{k} ? y_{d}$ where $d \in \operatorname{Inp}$, define $v_{d}$ as an assertion for which there are marked conjuncts of post $(d)$ which imply $v_{d}$ then,

$* \underset{d \in I m p}{\mathrm{~V}} \mathrm{v}_{d}\left[\operatorname{expr} / y_{d}\right] \rightarrow \xi$.

s The input command $P_{k} ? y$ and the output command $P_{j}$ lexpr, taken from process $j$ and process $k$ respectively, are called a syntactic matching pair. A syntactic matching pair is also a semantic matching pair if there exists an execution in which a communication between the matching communication commands actually takes place. Using the ordinary proof system it is possible to distinguish which are the semantic matching pairs. 


$$
\begin{array}{lc}
P_{1}^{\prime}:: & P_{2}^{\prime}:: \\
\{i=0\} & \{j=0\} \\
a:[\text { true } \rightarrow x:=1 \square \text { true } \rightarrow x:=2] ; & d:[\text { true } \rightarrow y:=1 \square \text { true } \rightarrow y:=2 \square \text { true } \rightarrow y:=3] \\
\{x=1 \wedge i=0\} & \{j=0\} \\
b:<P_{2} ! x ; i:=1> & e:<P_{2} ? z ; j:=1> \\
\{i=1\} & \{z=1 \wedge j=1\} \\
c:<P_{1} ? x ; i:=2> & f:<P_{2} !(y+z) ; j:=2> \\
\{x=3 \wedge i=2\} & \{z=1 \wedge j=2\} \\
I \equiv i=j \wedge(i=2 \rightarrow x=y+z) &
\end{array}
$$

Figure 3. History Outline.

It follows from the rule that the possible values of an output expression - expr - after the communication must include all possible values of the (matching) input variables $-\overrightarrow{y_{d}}-$ after communication. The reason for referring to the value of the output expression after communication is due to the fact that it may not take place.

Example: Assume $P_{j}$ !expr has exactly two semantic matching input commands, $e: P_{k} ? v$ and $f: P_{k} ? w$. Let $\operatorname{post}(e) \equiv\{(\nu=3 \vee 4) \wedge(w=7)\} \quad$ and $\quad p o s t(f) \equiv$ $\{(\nu=1 \vee 2) \wedge(w=5 \vee 6)\}$, and assume the first conjunct of post $(e)$ and the second conjunct of post $(f)$ are marked, then $v_{e} \equiv\{v=3 \vee 4\}, \quad v_{f} \equiv\{w=5 \vee 6\} \quad$ and $v_{e}[\operatorname{expr} / v] \vee v_{f}[\operatorname{expr} / w] \equiv\{\operatorname{expr}=3 \vee 4 \vee 5 \vee 6\}$.

\# Fifth rule (input): Let $c$ be a label of an input command $c: P_{k} ? y$. Let Out be the set of all labels of output commands which semantically match with that input command. For each output command $d: P_{j} ! \operatorname{expr} r_{d}$ where $d \in O u t$, define $v_{d}$ as an assertion for which there are marked conjuncts of post $(d)$ which imply $v_{d}$ then,

* $\underset{d \in \text { Out }}{\mathrm{V}} v_{d}\left[y / \operatorname{expr}_{d}\right] \rightarrow \xi$.

It follows from the rule that the possible values of an input variable $-y$ - after communication include all possible values of the (matching) output expressions $-\exp \vec{r}_{d}-$ after communication.

Example: Assume $c: P_{k}$ ? $y$ has exactly two semantic matching outputs commands, $e: P_{j} ! 3$ and $f: P_{j} !(v+w)$. Let post $(e) \equiv\{$ true $\}$ and post $(f) \equiv\{(v=1 \vee 2) \wedge(w=3 \vee 5)\}$ and assume it is marked, then $v_{e} \equiv\{3=3\}, v_{f} \equiv\{v+w=4 \vee 5 \vee 6 \vee 7\}$ and $v_{e}[y / 3] \vee v_{f}[y / v+w] \equiv\{y=3 \vee 4 \vee 5 \vee 6 \vee 7\}$.

The history outline concept as described above is not complete, in the sense that it is not possible to capture all possible information induced by a given history. First $\Theta_{h}(c)$ does not reflect the relative order in which the local states appear. Second, the pre assertion of an output command may not be as strong as possible. This may happen when an input command semantically matches with several output commands. In this case, we might want to capture the fact that at least one of those outputs has to send a specific value. Such a fact can not be expressed in a h.o. as defined above. It seems that by wider use of auxiliary variables, this information might also be expressed.

Example 5: A correct h.o. for the bracketed program $P^{\prime}$ and history $h_{1}$ from example 4 is shown in figure 3 .

Explanation: All conjuncts which refer to auxiliary variables are marked using the first rule, where as $P O$ we use the proof outline presented in example 3. 
This $P O$ provides also a proof of the invariant $I$. Marking the rest of the conjuncts of assertions from $P_{1}^{\prime}$ are done by using the third rule (with history $h_{1}$ ). The conjunct $(z=1)$ of post $(e)$ is marked by using the fifth rule while the conjunct $(z=1)$ of post $(f)$ is marked using the second rule.

Remark: In the above example, the history $h_{1}$ of program $P$ (example 2 ) is only a partial history of $P$, in that it does not refer to the auxiliary variables (a.v.). This should not cause any problem because any history can be uniquely extended to refer also to the exact values of the a.v. in each state of the sequence of states which form that history. This follows immediately from the role of the a.v. as history variables. In practice, as in the above example, it is not necessary to establish the extended history, because the conjuncts which refer to the a.v. can be marked using the first rule. To avoid cumbersome presentation we use $h$ for both the history without references to a.v. and its unique extension. The actual use can be understood from the context.

For the rest of the section we refer to an annotated program, where the assertions form a correct history outline with respect to a given history $h$ of process $i$. The previous notations post $\left(l_{i}\right)$ and post $(l c)$ refer now to that annotated program.

Lemma 2: For a history outline as defined above and for any $n$-tuple of labels $l c$, post (lc) is a true predicate after $l c$ for all traces which match with $h$.

Proof : omitted.

The following observation follows directly from the definition of a history location predicate $-S h_{l c}-$ (see the end of section two).

Observation: Let $h$ be the history of process $i$. If $h \rightarrow$ after $(c)$ and $l_{i} \neq c$ then $S h_{\left(l_{1}, \ldots, h_{h}\right)} \equiv$ false.

Lemma 3: For any location set $T_{l c}$ and history $h$, the associated history location predicate $S h_{l c}$ implies post $(l c)$. proof: $S h_{l c}$ is, by definition, the strongest predicate known to be true after $l c$ when only $h$ is known, while from lemma 2 , post $(l c)$ is a true predicate after lc for all traces which match with $h$.

Theorem 3: If $c$ is a label of process $i$, $h \rightarrow$ after $(c)$, and $\Phi(c)$ is the disjunction of all assertions post $\left(l_{1}, \ldots, l_{n}\right)$ such that $l_{i} \equiv c$, then $K H_{i}^{h}(\Phi(c))$.

Proof :

(1) $h \rightarrow$ after (c).

[Assumption]

(2) $\forall l c . S h_{l c} \rightarrow$ post $(l c)$.

[Lemma 2]

(3) $\forall l c .($ post $(l c) \wedge$ after $(c)) \rightarrow \Phi(c)$. [Def. of $\Phi(c)$ ]

(4) $\forall l c . S h_{l c} \rightarrow($ post $(l c) \wedge$ after $(c))$. [(1),(2),Obs.]

(5) $\forall l c . S h_{l c} \rightarrow \Phi(c)$.

(6) $K H_{i}^{h}(\Phi(c))$.

[(5), Def. of history knowledge]

[]

Now suppose it is to be proved that $K H_{i}^{h}(b)$ is true for a given program $P$ and that $h \rightarrow$ after $(c)$. The proof involves four steps similar to those used for location knowledge.

(1) A "strong enough" h.o. (with respect to $h$ ) is established for $P^{\prime}$.

(2) Assertion $\Phi(c)$, as defined above, is constructed. [by Theorem $3 K H_{i}^{h}(\Phi(c))$ ].

(3) It is proved that $\Phi(c)$ implies $b$. [by property $1 K H_{i}^{h}(b)$ ].

(4) By applying the auxiliary variables rule, ${ }^{6}$ $K H_{i}^{h}(b)$ is proved.

Example 6: Consider examples 2 \& 4. We prove $K H_{1}^{h_{1}}(y=2)$.

step 1: See h.o. presented in example 5.
step 2: $\Phi(c) \equiv$ post $\left(c\right.$, init $\left._{2}\right) \vee$ post $(c, d) \vee$ post $(c, e) \vee$ post $(c, f)$ $\equiv$ false $\vee$ false $\vee$ false $\vee$ $(x=3 \wedge z=1 \wedge i=j=2 \wedge I \wedge$ after $(c, f))$.

${ }^{6}$ An auxiliary variable rule similar to the one presented in the previous section is used. 
step 3: $\Phi(c)$ implies $y=2, \quad$ by property 1 $K H_{1}^{h_{2}}(\mathrm{y}=2)$.

step 4: By applying the auxiliary variable rule, $K H_{1}^{h_{1}}(y=2) \quad$ ․

It is easy to modify the proof method just presented to prove knowledge assertions when only a partial history is given. The modification which is needed is in how a correct (partial) h.o. is established in such cases. This will involve redefining the third marking rule, which showed how to satisfy proof obligations directly from the history. Now only a partial history can be used.

As an example let us modify the proof method, so as to prove state knowledge assertions. Let $s$ denote the given state. The modified third marking rule takes the following form:

\# Third rule: * $\quad s \rightarrow(\xi \wedge$ after $(c))$.

Defining the (usual) four step proof method is now obvious.

In general if the current location is not given as part of the partial history then process $i$ can know the disjunction of $\Phi(c)$ over all its possible locations.

Note that now it will make sense to mark preconditions of statements on the basis of the marking of its postcondition (in the process whose partial history is given), at least back to an input/output statement. Previously there was no need to do such 'backward marking' since the entire history was available.

Modifying the proof method for proving location knowledge assertions, involves omitting the third marking rule completely. Note that in that case, as expected, the resulting marking rules ${ }^{7}$ define what has previously been defined as a location outline.

\section{NESTED KNOWLEDGE}

An extension of the knowledge definition and the proof method to deal with knowledge about knowledge is natural. In order to prove an assertion like: process $i$ knows after $c$ that process $j$ knows $p$, without further information about the exact location of process $j$ $\left(K L_{i}^{c}\left(K L_{j}(p)\right)\right)$, one must prove that process $j$ knows $p$ at all locations possible when process $i$ is after $c$. That is for every label $a$ of process $j$ either $K L_{i}{ }^{c}(\neg a f t e r(a))$ or $K L_{j}^{a}(p)$. Again, considering example 2, such an assertion is: $K L_{2}^{e}\left(K L_{1}(x=z)\right)$.

More generally, we consider a claim of the form $K H_{i}^{h}(b)$ where $b$ may include other knowledge operators (but is still non-temporal). The assertion $b$ often (but not necessarily) will include the form $K H_{j}(p)$, without a specific history as a parameter. This means that for every local history $h^{\prime}$ of process $j$ which matches with one of the possible traces of the system, $K H_{j}^{h^{\prime}}(p)$. It is also possible to consider history knowledge with only partial histories. For example, $K H_{j}^{s}(p)$ ( $s$ - for state) means that process $j$ will have available a local history $h^{\prime}$ which can be used to show $p$ true, but $h^{\prime}$ is not given as a parameter. Thus, $h^{\prime}$ may be any history of process $j$ which matches with a trace of the system and also is consistent with the state $s$. Note that, at least when appearing in the scope of an outer knowledge operator, this differs from $K S_{j}^{s}(p)$, where process $j$ does not have a local history available.

Let us concentrate, as an example, on assertions of the form $K H_{i}^{h}\left(K H_{j}(p)\right)$, where $p$ does not contain knowledge operators. This means that, given local history $h$, process $i$ knows that process $j$ (history) knows $p$, without further information about the actual history of process $j$. Thus this assertion is true only if process $j$ knows $p$ with any of its possible histories. Although process $i$ cannot in general know the entire actual local history of process $j$, it can know (by using $h$ ) various assertions which will be true of any local history of process $j$ which is consistent both with the possible traces of the system and with $h$. Such assertions can be proven by devising a history outline for $h$, and properly marking all of the conjuncts as described previously. This will be in fact the first stage in proving

\footnotetext{
${ }^{7}$ actually the first marking rule is enough.
} 
$K H_{i}^{h}\left(K H_{j}(\mathrm{p})\right)$. The following claim is then crucial to the rest of the proof method: informally, the actual local history of process $j$ must satisfy all marked assertions annotated in process $j$, from (any) history outline established with respect to the above $h$. The following lemma express this idea formally.

Let $h$ and $h^{\prime}$ denote for the rest of the section, local histories of processes $i$ and $j$ respectively. Let post $_{k}(c)$ denote a post assertion of a command labeled by $c$, which can appear in some history outline established with respect to history $h$, and recall the definition of $\Theta_{h}(c)$ from the previous section.

Definition: Two histories $h$ and $h^{\prime}$ as above, are consistent iff there exists a trace $t$ such that both $\operatorname{match}(t, h)$ and match $\left(t, h^{\prime}\right)$ hold.

Lemma 4: If two histories $h$ and $h^{\prime}$ as above, are consistent then for each label $c$ of process $j$, $\Theta_{h^{\prime}}(c) \rightarrow \operatorname{post}_{h}(c)$.

\section{Proof : Omitted.}

Next we describe how to establish the second stage in proving a nested knowledge assertion, again using marking rules. We require a (single) history outline which will be correct with respect to every history of process $j$ which is consistent with the given history $h$ of process $i$. The aim of this is to be able to show $K H_{j}(p)$ in context. Its correctness will follow from lemma 4. The intuition is to allow marking the assertions annotated in process $j$ if they can appear in the history outline (with respect to the above $h$ ) from the first stage. Formally, the assertions of the annotated program in the new proof form a correct (multi) history outline as described above, if they can be marked using the marking rules from the previous section, where the third rule is replaced by:

\# Third rule : Let $c$ be a label of process $j$, and let $\operatorname{post}_{h}(c)$ denote, an assertion from a history outline previously established with respect to $h$.

$$
\text { * } \operatorname{post}_{h}(c) \rightarrow \xi \text {. }
$$

Now, once such a (multi) history outline is established, we can deduce from it the truth of the needed assertion in the same manner as described in the beginning of this section for location knowledge.

More formally, let $\Phi(a)$ be defined as before but with respect to the history outline just described. From theorem 3 and lemma 4, it follows that for every history $h^{\prime}$ (of process $j$ ) consistent with the above $h$ such that $h \rightarrow \operatorname{after}(a), K H_{j}^{h^{\prime}}(\Phi(a))$. Thus, in order to prove $K H_{i}^{h}\left(K H_{j}(p)\right)$, one must prove that for every label $a$ of process $j$, either $K H_{i}^{h}(\neg a f t e r(a))$ or $\Phi(a) \rightarrow p$.

In general, for $K H_{i}^{h}(b)$, the proof of $\mathrm{b}$ will be as previously described, except that certain conjuncts can be marked immediately if they define assertions true of any history, state, or location which is available in $b$ and is consistent with $h$. This process can be continued recursively, for an arbitrarily deep nesting of knowledge operators.

Example 7: Consider, yet again, the program from the previous examples. Using the technique described above, we can easily show that $K H_{1}^{h_{1}}\left(K H_{2}(x=3)\right)$.

From the history outline established in example 5, it follows that $K H_{1}^{h_{1}}\left(\neg a f: e r\left(\right.\right.$ init $\left.\left._{1}\right)\right)$, $K H_{1}^{h_{1}}(\neg a f t e r(d))$, and $K H_{1}^{h_{s}}(\neg a f t e r(e))$. Next a correct history outline with respect to all histories of process $j$ consistent with $h_{1}$ is established. This is done by adding $y=2$ as a conjunct of post $(f)$ and retaining only those conjuncts in process $P_{1}$ which refer to auxiliary variables. With respect to that history outline, $\Phi(f) \rightarrow(x=3)$. [0

\section{CONCLUSIONS}

In this paper we have shown how to prove knowledge assertions under a variety of knowledge assumptions about the available local information, assuming the text of the program as global information. We have generalized existing proof techniques, by relaxing the proof obligations for assertions which follow from the given knowledge assumption, and shown 
what knowledge follows from such a proof.

The motivation for this work is in the specification and design of distributed programs, and in future work we plan to demonstrate the utility of knowledge for a variety of tasks.

Acknowledgement: We would like to thank Nissim Francez for helpful discussions on the subject.

\section{REFERENCES}

[AFR] Apt,K.R., Francez, N., and de Roever, W.P. A proof system for communicating sequential processes, ACM-TOPLAS, 2,3 1980, 359 . 385.

[Ap] Apt,K.R., Formal justification of proof system for communicating sequential processes. JACM 30,1 1983, 197-216.

[B] Barringer, H. A survey of verification techniques for parallel programs, LNCS 1911985.

[BHR] Brookes, S.D., Hoare C.A.R., Roscoe A.W. A theory of communicating sequential processes, JACM 31,3 1984, 560-599.

[CM] Chandy, M., and Misra, J. How processes learn, $A C M-P O D C$ 1985, 204-214.

[FHV] Fagin, R., Halpern, J., and Vardi, M. A model theoretic analysis of knowledge, IEEE-FOCS 1984, 268-278.

[HF] Halpern, J., and Fagin, R. A formal model of knowledge, action, and communication in distributed systems: preliminary report, $A C M-P O D C$ 1985, 224-236.

[HM1] Halpern, J., and Moses, Y. Knowledge and common knowledge in a distributed environment, ACM-PODC 1984, 50-61.

[HM2] Halpern, J., and Moses, Y. A guide to the modal logic of knowledge and belief, IJCAI 1985.

[Le] Lehmann, D. Knowledge, Common Knowledge and related puzzles, $A C M-P O D C$ 1984, 62-67.

[Ow] Owicki, S. Axiomatic proof technique for parallel programs, Computer Science Dept., Cornell University, Ph.D. thesis, 1975.

[OG] Owicki, S., and Gries, D. An axiomatic proof technique for parallel programs, I. Acta Inf. 6, 1976, 319-340.
[OL] Owicki, S., Lamport, L. Proving liveness properties of concurrent programs, ACM-TOPLAS 4,3 1982, 455-495.

[LSP] Lamport, L., Shostak, R., Pease, M. The Byzantine General Problem, ACM-TOPLAS 4,3 1982, 382-401.

[M] Misra, J. Reasoning about network of communicating processes, Proc. Advanced NATO Institute on Logic and Models for Verification and Specification of Concurrent Systems, Oct. 1984.

[PR] Parikh, R., and Ramanujam, R. Distributed processes and the logic of knowledge: preliminary report, $L N C S$ 193, 256-268. 